\title{
Sleeve gastrectomy leaks: the way we treat it
}

\section{Introduction}

Laparoscopic vertical sleeve gastrectomy (LVSG) has become a standard procedure in the bariatric surgeons' armamentarium for obesity surgery. As the number of cases increase, so do the number of complications. Staple line leak can be a devastating morbidity, which can consume health care resources as well as be detrimental to the patient ${ }^{1-6}$ just as the technique for the LVSG has evolved, so have the treatment methods for the management of post-operative leak (Figure $1)$.

According to the International sleeve gastrectomy expert panel consensus statement from Rosenthal in 2012, initial intervention included surgical exploration, drainage; esophageal stenting and possible conversion to Roux-en-Y bypass. ${ }^{1}$ Several studies have shown improvement of acute and chronic LVSG leaks with endoscopic stenting. ${ }^{7-9}$ Over-the-scope endoscopic clips and endoscopic suturing are new techniques not part of the algorithm that are becoming more widely used with positive results. ${ }^{10-13}$ When other conservative methods have failed, RYGB or Roux-en-Y fistula jejunostomy have also been proposed as alternative treatments for non-healing gastric leaks. ${ }^{9}, 14-17$ With the increasing experience of surgeons performing LVSG and treating complications, Nedelcu has recently proposed an algorithm for treatment based on size of the leak and stenosis of the sleeve. ${ }^{18}$ The aim of this study was to review our experience and outcomes of LVSG treatment and design an algorithm of successful treatment.

Approval for this study was granted by the Banner Health institutional review board. Patients were identified from the authors' prospectively maintained bariatric database. All patients between 2012 and 2014 were reviewed, and 11 patients were identified and treated at our institution. Data collected included patient demographics, operative, endoscopic and medical interventions, and follow-up outcomes. Patient demographics included age, sex, body mass index (BMI), co-morbidities, number of days from initial surgery until recognition of leak. Operative data included location of initial surgical intervention, need for repeat interventions and type, placement of feeding jejunostomy tube. Endoscopic interventions included use of OVESCO ${ }^{\circ}$ over the scope clip, Boston Scientific fully covered WallFlex ${ }^{\mathrm{TM}}$ esophageal stent, Apollo Over Stitch ${ }^{\mathrm{TM}}$. Post-operative data included need for multiple interventions, nutritional support, and number of days until resolution of leak.

During the study period from 2012-2014, 556 patients underwent LVSG for the treatment of morbid obesity. We identified a total of 8 LVSG leaks (1.4\%). Three patients did not have initial surgery at our institution and were transferred from an outside hospital for further treatment.

Mean age of the patients was 40.7(25-53). Of these 11 patients, $9(81 \%)$ were women. Mean BMI on the day of surgery was $45.3 \mathrm{~kg} / \mathrm{m}^{2}$ (26.2-61.7). The average number of days to recognition of LVSG leak was 19.8 (4-49). The average number of days to resolution of LVSG leak was 126 (13-503). Ten (91\%) of the patients have had resolution of their gastric sleeve leak. One ( $9 \%)$ patient at the time of completion

\author{
Volume 2 Issue 5 - 2015
}

\author{
Flavia C Soto, Albert Chen, jill Gorsuch, Emil \\ Graf, David Podkameni \\ Clinical Assistant Professor in the Department of Surgery, \\ University of Arizona College of Medicine, USA
}

Correspondence: Flavia C Soto, Clinical Assistant Professor in the Department of Surgery, University of Arizona College of Medicine, US 601900 N. Higley Road Gilbert, Arizona 85234, USA,Tel 9545992153,Email Flavia.Soto@bannerhealth.com

Received: May 08, 2015 | Published: July 02, 2015

of the study was still undergoing treatment for an unresolved closure of his leak.

According to the definition from Rosenthal et al., ${ }^{1}$ acute leak (within 7days of surgery) were recognized in 2(18\%) patients. Early leaks (within 1-6weeks from surgery) were recognized in $9(81 \%)$ patients. There were no late (greater than 6 weeks from surgery) or chronic leaks (greater than 12weeks from surgery).

Interventional radiological (IR) drainage occurred in 5(45\%) patients. Eight $(72 \%)$ of the patients had a surgical intervention. Laparoscopic washout and drain placement occurred with 4(36\%) of the patients. Re-operation was required in $4(36 \%)$ of the patients. Two (18\%) patients underwent an exploratory laparotomy with jejunoserosal patch. One of the two had to be taken back postoperative day 1 due to small bowel obstruction in the afferent limb of the jejunoserosal patch. One ( $9 \%$ ) patient underwent a splenectomy for chronic splenic abscess secondary to the gastric fistula. One underwent exploratory laparotomy, washout of peritoneal cavity and drainage placement for dislodged stent causing dehiscence of gastric staple line then required re-suturing of the greater curvature of the sleeve with running PDS suture.

Nutritional support was supplied by feeding jejunostomy in 4 $(36 \%)$ patients, total parenteral nutrition (TPN) in $3(27 \%)$ patients, and a combination of TPN and feeding jejunostomy in $3(27 \%)$ patients. Four (36\%) patients had an Ovesco Over-The-Scope Clip ${ }^{\circledR}$ used to close the gastric fistula. This was successful as a stand alone intervention in $1(9 \%)$ of the four patients attempted. The other three $(27 \%)$ were in combination with other therapies, refer to demographic table, patients: $4,5,10$. One $(9 \%)$ patient had fibrin glue injected into the fistula as a combination treatment with other therapies, (patient 5). Three (27\%) patients had Boston Scientific Fully Covered WallFlex ${ }^{\mathrm{TM}}$ Esophageal Stents placed. All three of the patients with stents were used in conjunction with other therapeutic modalities (Patients 9-11). Apollo OverStitch ${ }^{\mathrm{TM}}$ of the fistula was used in $3(27 \%)$ patients in combination with other therapeutic modalities (patients 9-11).

Figure: Algorithm for Treatment of Sleeve Gastrectomy Leaks 


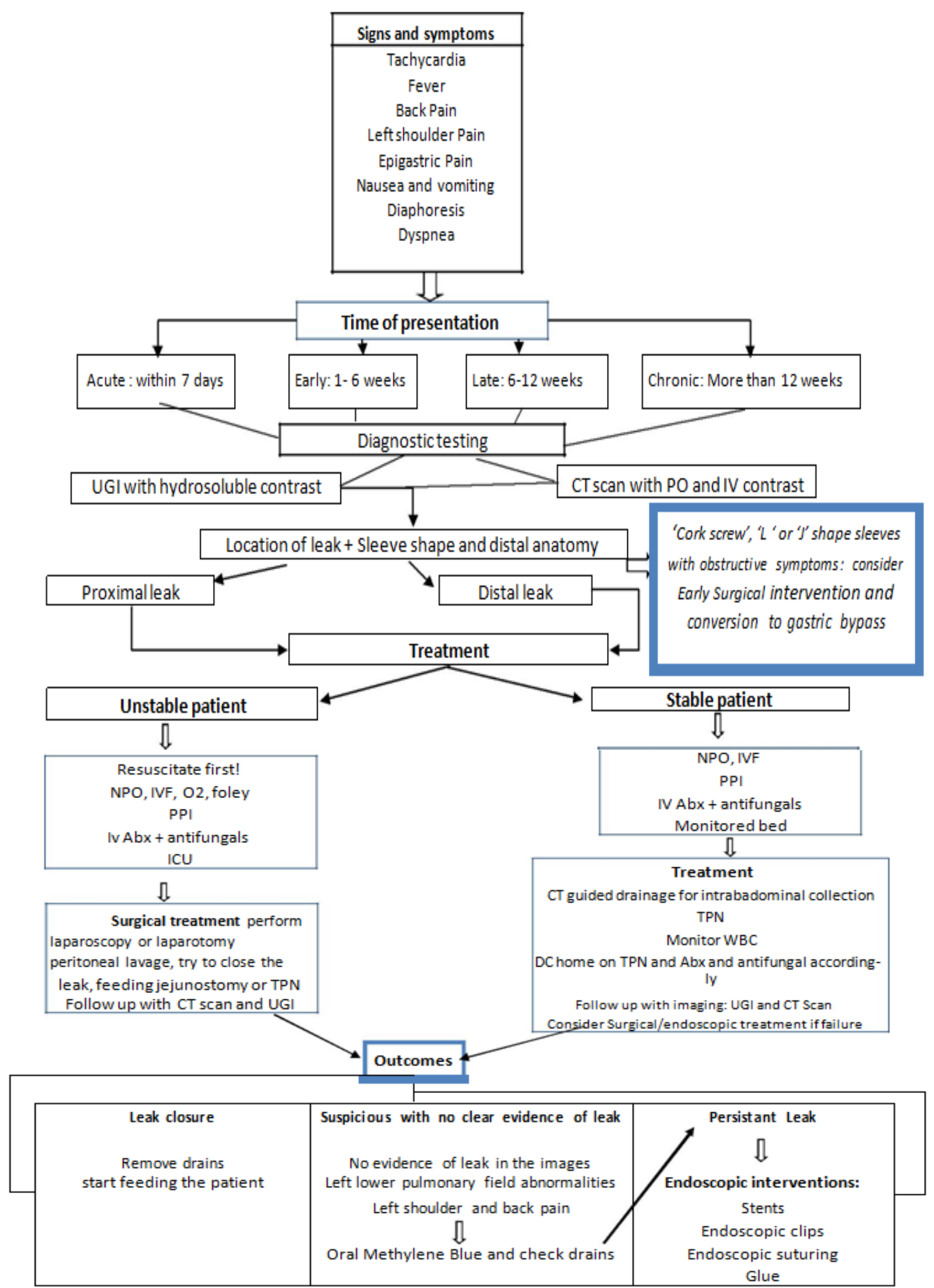

Figure I Algorithm for Treatment of Sleeve Gastrectomy Leaks 


\section{Conclusion}

Overall, early recognition and high suspicion for LVSG leak is the first step to successful treatment and prevention of long term morbidity and mortality. Initial recognition should include use of CT scan, upper GI radiologic evaluation and EGD to identify not only the leak but the anatomy of the sleeve.

Once a leak is recognized, one must consider the timing of the leak, and the initial ability to provide conservative management with drainage when appropriate based on clinical stability of the patient, nutritional support and endoscopic intervention. If conservative management is not successful, surgical approach is the answer.

\section{Acknowledgements}

None.

\section{Conflict of interest}

The author declares no conflict of interest.

\section{References}

1. Rosenthal R, Diaz AA, Arvidsson D, et al. International Sleeve Gastrectomy Expert Panel Consensus Statement: best practice guidelines based on experience of 12,000 cases. Surg Obes Relat Dis. 2012;8(1):819.

2. Sakran NG, Goitein D, Raziel A, et al. Gastric leaks after sleeve gastrectomy: a multicenter experience with 2,834 patients. Surg Endosc. 2013;27(1):240-245.

3. Aurora AK, Khaitan L, Saber AA. Sleeve gastrectomy and the risk of leak: a systematic analysis of 4,888 patients. Surg Endosc. 2012;26(6):15091515.

4. Bellanger DE, Greenway FL. Laparoscopic Sleeve Gastrectomy, 529 Cases without a Leak: Short-Term Results and Technical Considerations. Obes Surg. 2011;21(2):146-150.

5. Bekheit MK, Khaled Katri M, Wael Nabil, et al. Earliest signs and management of leakage after bariatric surgeries: Single institution experience. Alexandria Journal of Medicine. 2013;49(1):29-33.

6. Baker RF, Foote J, Kemmeter P, et al. The Science of Stapling and Leaks. Obes Surg. 2004;14(10):1290-1298.
7. Alazmi W, Al-Sabah S, Ali DA, et al. Treating sleeve gastrectomy leak with endoscopic stenting: the kuwaiti experience and review of recent literature. Surg Endosc. 2014;28(12):3425-3428.

8. Abraham AR, Kaleem Rizvon, Jaspreet Singh, et al. Successful management of a gastric sleeve leak with an endoscopic stent. Case Reports in Gastrointestinal Medicine. 2012:1-4.

9. Chouillard EC, Chahine E, Schoucair N, et al. Roux-En-Y FistuloJejunostomy as a salvage procedure in patients with post-sleeve gastrectomy fistula. Surg Endosc. 2014;28(6):1954-1960.

10. Iacopini FL, Di Lorenzo N, Altorio F, et al. Over-the-scope clip closure of two chronic fistulas after gastric band penetration. World $J$ Gastroenterol. 2010;16(13):1665-1669.

11. Dakwar AS, Ahmad Assalia, Iyad Khamaysi, et al. Late Complication of Laparoscopic Sleeve Gastrectomy. Case Reports in Gastrointestinal Medicine. 2013:1-5.

12. Kirschniak AK, Kratt T, Stüker D, et al. A new endoscopic over-thescope-clip system for the treatment of lesions and bleeding in the GI tract: first clinical experiences. Gastrointest Endosc. 2007;66(1):162167.

13. Seebach L, Bauerfeind P, Gubler C. "Sparing the surgeon": clinical experience with over-the-scope clips for gastrointestinal perforation. Endoscopy. 2010;42(12):1108-1111.

14. Thompson CA, Ahmad H, Lo Menzo E, et al. Outcomes of laparoscopic proximal Gastrectomy with esophagojejunal reconstruction for chronic staple line disruption after laparoscopic sleeve gastrectomy. Surg Obes Relat Dis. 2014;10(3):455-459.

15. Nedelcu AS, Skalli M, Deneve E, et al. Surgical management of chronic fistula after sleeve gas. Surg Obes Relat Dis. 2013;9(6):879-884.

16. van de Vrande S, Himpens J, El Mourad H, et al. Management of chronic proximal fistulas after sleeve gastrectomy by laparoscopic Roux-limb placement. Surg Obes Relat Dis. 2013;9(6):856-861.

17. Moon RS, Shah N, Teixeira AF, et al. Management of staple line leaks following sleeve gastrectomy. Surg Obes Relat Dis. 2015;11(1):54-59.

18. Nedelcu M. Outcomes of Leaks after Sleeve Gastrectomy Based on a New Algorithm Addressing Leak Size and Gastric Stenosis. Obes Surg. $2015 ; 25(7): 1258-1260$. 